\title{
Modeling of Needle-Tissue Interaction Forces During Surgical Suturing
}

\author{
Russell C. Jackson and M. Cenk Çavuşoğlu
}

\begin{abstract}
This paper presents a model of needle tissue interaction forces that a rigid suture needle experiences during surgical suturing. The needle-tissue interaction forces are modeled as the sum of lumped parameters. The model has three main components; friction, tissue compression, and cutting forces. The tissue compression force uses the area that the needle sweeps out during a suture to estimate both the force magnitude and force direction. The area that the needle sweeps out is a direct result of driving the needle in a way that does not follow the natural curve of the needle. The friction force is approximated as a static friction force along the shaft of the needle. The cutting force acts only on the needle tip. The resulting force and torque model is experimentally validated using a tissue phantom. These results indicate that the proposed lumped parameter model is capable of accurately modeling the forces experienced during a suture.
\end{abstract}

\section{INTRODUCTION}

Even with the assistance of robotic surgical systems, suturing is a challenging and time consuming task during Minimally Invasive Surgery (MIS). Therefore, automating the suturing task is desirable. The robot pre-plans the suture motion, which when combined with force feedback, will allow the robot to minimize any tissue trauma that might occur as a result of suturing. In order for the robot to successfully complete an automated suture, the robot must understand the types of forces and torques that a needle experiences during a typical suture.

When a surgeon drives a needle through tissue during suturing, he inserts the needle with the tip normal to the tissue surface. The needle path also follows the curve of the needle [8]. Following these guidelines reduces the tissue trauma and aids healing. During a surgical suture, the surgeon can re-grasp the needle as necessary. He can even re-grasp the needle through the wound that is being sutured. Laparoscopic sutures are more difficult due to the reduced dexterity of the surgeon (there are only 4 degrees of freedom available due to the instrument portal). Despite the reduction in dexterity, surgeons still adhere to the same principles of needle driving as they would in an open suture. This can be fatiguing for the surgeons due to the combination of repetition required with suturing and the difficulties associated with the reduced degrees of freedom. One method of increasing the dexterity of a laparoscopic suture is to use a robotic assistant such as the daVinci ${ }^{\circledR}$ system (Intuitive Surgical, Sunnyvale, California). Even though the daVinci ${ }^{\circledR}$ robot improves the surgeon's

This work was supported in part by NSF under grants IIS-0805495, IIS0905344, and CNS-1035602, and National Institutes of Health under grant R21 HL096941.

The authors are with the department of Electrical Engineering and Computer Science (EECS) at Case Western Reserve University in Cleveland, OH. The corresponding author M. Cenk Çavuşoğlu can be reached at cavusoglu@case.edu dexterity, the surgeon must still complete the entire suture manually. A robot that can intelligently drive a suture needle could reduce surgeon fatigue while increasing dexterity. In addition to reducing the surgeon's fatigue, automated suturing also has the potential to improve the speed of the suture. This could significantly decrease operation times and consequently improve the patient's post operative outlook.

The goal of this paper is to analyze the interaction forces experienced when a rigid curved suture needle is driven through a tissue sample. This includes modeling the forces and torques generated during a suture using a computationally efficient lumped parameter model. Previous studies on the different techniques that are used to model tissue needle forces are discussed in section II. This is followed in section III by a discussion of the needle motion geometry and how that might impact the forces that the needle could sense as it cuts the tissue. Next, the lumped models that are used to describe the tissue forces are developed in section IV. Experimental validation of the needle force models with a detailed analysis and evaluation of the needle force models is presented in section $\mathrm{V}$. This paper concludes with the final comments and outline future work that is planned in section VI.

\section{NeEdle Force Modeling}

There has been significant work on modeling the needle tissue interaction forces during either straight or flexible needle insertion [1]. The purpose of many of these different models are for brachytherapys involving the precise placement of radioactive beads that will irradiate the surrounding tissue and kill any nearby cancerous cells. For example, Chentanez et al. have modeled the tissue deformation of the prostate gland during the insertion of a straight hollow needle [3]. The modeling is performed using a three dimensional Finite Element Model (FEM) where the element mesh updates dynamically as necessary. This can be a very accurate method for modeling both material deformation and the forces generated during the deformation. One disadvantage of using a complex three dimensional FEM is that it can be difficult to solve the FEM in real-time such as would be needed for an automated needle path plan. Altervotiz et al. have worked on similar modeling of the prostrate gland [2]. Their models use a two dimensional FEM instead of a three dimensional one. This is one way of improving computational efficiency at the cost of model accuracy. There have been many papers published that use FEM for modeling needle tissue interaction forces. Since material properties important to the FEM calculations can vary significantly between tissue types, Maghsoudi et al. publish a work that 
analyzes the sensitivity of the FEM algorithm to parameter deviation. This could include properties such as Young's Modulus and the Poisson Ratio [5]. A significant amount of the force that a needle experiences is concentrated at the tip. This means that it is important to model the tearing event that the tissue undergoes [6]. Okamura et al. uses a lumped force model to simulate the axial forces a straight rigid needle would experience when it is inserted into a liver [7]. In the lumped force model each $f$ represents a contribution to the net force from a different source.

$$
f_{\text {needle }}(x)=f_{\text {friction }}(x)+f_{\text {cutting }}(x)+f_{\text {stiffness }}(x)
$$

Compared to FEM based analysis, the lumped needle force is computationally efficient. A detailed analysis of needle tissue interaction forces sensed during surgical suturing is unavailable in the literature. Many of the previous works model the forces that are experienced by needles used for biopsies and therapeutic applications. These needles are long, straight, hollow, and potentially flexible. The needles used for performing a suture are short, curved, rigid and solid. Since suture needles are inflexible, they will not comply with the tissue during a suture. Most lumped models available in the literature do not model off axial forces resulting from tissue displacement. This force is very critical for suture applications as will become evident in the following analysis (e.g. Fig. 9). Also FEM models are computationally intensive and therefore, are not suitable for use as a component of an inline needle control scheme.

\section{Suture NeEdle Motion MOdel}

The suture needle is approximated as a circular arc [8]. The canonical motion of the needle is shown in Fig. 1. This figure is drawn with respect to the geometric center of the needle $(C)$. The canonical motion can be expressed with two components. The first component is a rotation about the center of the needle $(\omega)$. The second component is the velocity ( $\mathbf{v}$ ) of the geometric center of the needle. The radius of the needle is defined as $r$. The coordinate frame defined by by $x_{f}$ and $y_{f}$ is the coordinate frame of the force and torque measurement. This frame is attached to the base of the needle, but is aligned to the world frame, $x_{w}$ and $y_{w}$. The tissue corresponds to the shaded region.

\section{A. Ideal Needle Motion}

Since it has been established that the best sutures are those that follow the natural curvature of the needle [8], the ideal motion of the needle is to move in a circular arc about the center. This motion is shown in Fig. 2(a). This motion plan reduces the velocity $\mathbf{v}$ to zero and the needle simply rotates with a constant speed $(\omega)$ about the point $O$. In this case, $O$ is aligned with the needle center $(C)$. This needle motion is naturally planar.

\section{B. Non Ideal Needle Motion}

When a robot tries to perform an ideal needle motion, uncertainty in the needle mount would result in non-ideal needle motions. As the needle is assumed to be rigid, the

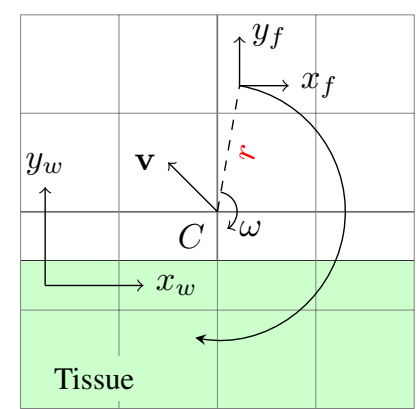

Fig. 1: Canonical Needle Motion. The needle rotates with an angular velocity of $\omega$. At the same time the geometric center $(C)$ has a velocity of $\mathbf{v}$. The tissue is indicated by the green shaded region.

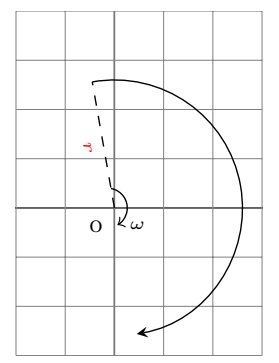

(a) Ideal

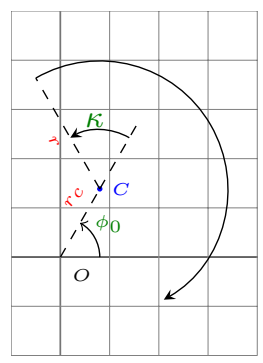

(b) Non-Ideal
Fig. 2: (a) Ideal Motion Model. The needle rotates about the center $(O)$ in which the velocity $\mathbf{v}$ is 0 . (b) Non-Ideal Motion Model. The center, $C$, rotates about the center of motion $(O)$ with a radius $r_{c}$. The starting angle $\left(\phi_{0}\right)$ and the error angle $(\kappa)$ are both geometric properties of the system. This particular image exaggerates the magnitude of $r_{c}$ and $\kappa$.

non-ideal motion is related to the motion of the geometric center of the needle. Fig. 2(b) shows a snapshot of the motion. The center of the needle $(C)$ rotates about the center of motion $(O)$ with a radius $r_{c}$. The starting angle of the geometric center is the angle $\phi_{0}$. The angle $\kappa$ corresponds to the relationship between the angle of the base of the needle and the angle of the geometric center of the needle. As the needle is driven through the tissue, the movement of geometric center about $O$ will induce stress in the tissue due to the non tangential motion of the needle body. Even though the needle mount could include non planar errors that would affect the force profile, for the purposes of this paper non planar errors are ignored to simplify the analysis. As a result, the motion of the needle will be assumed to be planar. However, the proposed model and analysis is not inherently restricted to planar motions. The area that the needle sweeps will be incorporated into the lumped forces model.

\section{Area Sweep}

Following the natural path of the needle as in Fig. 2(a) minimizes the tissue stresses. If the suture does not follow the path of the needle, then the needle will sweep out an area as it moves through the tissue. Since the area sweep should 
be minimized, calculating the area swept by the needle could be a simple method of measuring the quality of the needle path.

The area swept by the needle can be modeled as shown in Fig. 3. The area of the parallelogram formed by the vectors $\mathrm{d} \ell$ and $\mathbf{v} \mathrm{d} t$ is the amount of tissue distortion that the needle is creating over a small time step $(\mathrm{d} t)$. The swept area can be computed as

$$
d a=\|\mathbf{v}\| \mathrm{d} t\|\mathrm{~d} \ell\| \sin \gamma,
$$

where $\gamma$ is the angle between the two vectors $\mathbf{v}$ and $\mathrm{d} \ell$ as shown in Fig. 3 . This area sweep has a direction that is outward normal to the needle curve. Alternatively, the area can be calculated using vector notation as

$$
\mathrm{d} \mathbf{a}=\mathbf{v}(\theta) \mathrm{d} t \times \mathrm{d} \ell(\theta),
$$

where $\theta$ is the angle of the needle segment. Since the motion is planar, only one directional component will be non zero $(z)$. This means that the magnitude of the area corresponds to the third component of the vector generated by the cross product. This allows a direction to be assigned to the area that the needle sweeps out. That is because the direction of the needle tissue compression must be known. The normal vector representation of the swept area is given by

$$
\mathrm{d} \mathbf{a}_{n}=\frac{\mathrm{d} \ell}{\|\mathrm{d} \ell\|} \times \mathrm{d} \mathbf{a}
$$

where the subscript $n$ indicates that the quantity is normal to the needle tangent in the $x-y$ plane. Using the motion model outlined in Fig. 2(b), it is possible to calculate the area swept out by the needle and its direction. The needle segment vector is based on the angle of the needle

$$
\mathrm{d} \ell=r\left[\begin{array}{c}
-\sin (\theta) \\
\cos (\theta) \\
0
\end{array}\right] \mathrm{d} \theta,
$$

The angle $\theta$ can be computed as follows:

$$
\theta=\phi+\kappa-\psi,
$$

where $\phi, \kappa$, and $\psi$, as shown in Fig. 3, are the angle of rotation of the needle center about the base frame, the angle offset of the needle base, and the position along the needle arc. The velocity of the needle segment can then be calculated as

$$
\begin{aligned}
\mathbf{v}(\phi, \theta, \omega) & =r_{c}\left[\begin{array}{l}
0 \\
0 \\
\omega
\end{array}\right] \times\left[\begin{array}{c}
\cos (\phi) \\
\sin (\phi) \\
0
\end{array}\right] \\
& +r\left[\begin{array}{l}
0 \\
0 \\
\omega
\end{array}\right] \times\left[\begin{array}{c}
\cos (\theta) \\
\sin (\theta) \\
0
\end{array}\right] .
\end{aligned}
$$

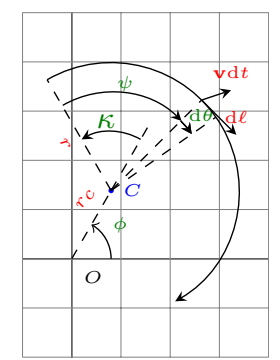

Fig. 3: The area swept out by a small segment of needle $\mathrm{d} \ell$ during an incremental motion $\mathbf{v d} t$.

Combining (3), (4), (5), and (7) and simplifying, yields

$$
\begin{aligned}
\mathrm{d} \mathbf{a}_{n} & =r \omega r_{c}\left[\begin{array}{c}
\cos (\theta) \\
\sin (\theta) \\
0
\end{array}\right] \cos (\phi) \sin (\theta) \\
& -r \omega r_{c}\left[\begin{array}{c}
\cos (\theta) \\
\sin (\theta) \\
0
\end{array}\right] \sin (\phi) \cos (\theta) .
\end{aligned}
$$

Notice that the area swept is only due to the motion of the geometric center. This is because the rotation of the needle about its center is always along its tangent. If $\phi=\theta$, then the area swept out by the segment is 0 . This means that there may exist a point on the needle that is not sweeping out any area. The integral of (8) over the needle arc and over time will give the total area swept out.

$$
\mathbf{a}_{n}=\int_{t_{0}}^{t_{1}} \int_{\theta_{0}(t)}^{\theta_{1}(t)} \mathrm{d} \mathbf{a}_{n}(\phi(t), \theta, \omega) \mathrm{d} \theta \mathrm{d} t
$$

The variables $t_{0}$ and $t_{1}$ are the experimental start and stop times respectively. $\theta_{0}$ and $\theta_{1}$ are the angles for which the needle is inside the tissue sample. The area swept $\mathbf{a}_{n}$ can be used to measure both the quality of a needle path and to estimate the force on the needle due to tissue deformations. The area swept by the needle is demonstrated in Fig. 4.

\section{Suture NeEdLE Forces}

The force and torque acting on the needle will be modeled as the sum of three lumped forces.

$$
\begin{aligned}
\mathbf{f}_{\text {needle }}(\phi(t)) & =\mathbf{f}_{\text {friction }}(\phi(t)) \\
& +\mathbf{f}_{\text {cutting }}(\phi(t))+\mathbf{f}_{\text {normal }}(\phi(t))
\end{aligned}
$$

\section{A. Friction Forces}

The friction force acting on the needle is a constant that acts in opposition to the needle motion over the entire length of the needle. As long as the velocity of the needle segments are approximated as constants, it is acceptable to use this models.

$$
\mathbf{f}_{\text {friction }}(\phi(t))=\int_{\theta_{0}(\phi)}^{\theta_{1}(\phi)}-\mu_{s} \mathrm{~d} \ell(\theta) \mathrm{d} \theta
$$

The variable $\phi(t)$ is the position of the needle center as a function of time $(t) . \theta_{0}$ and $\theta_{1}$ are the start and end angles of the needle in the tissue. $\mu_{s}$ is the friction coefficient. This sums the friction forces so that the force can be calculated 


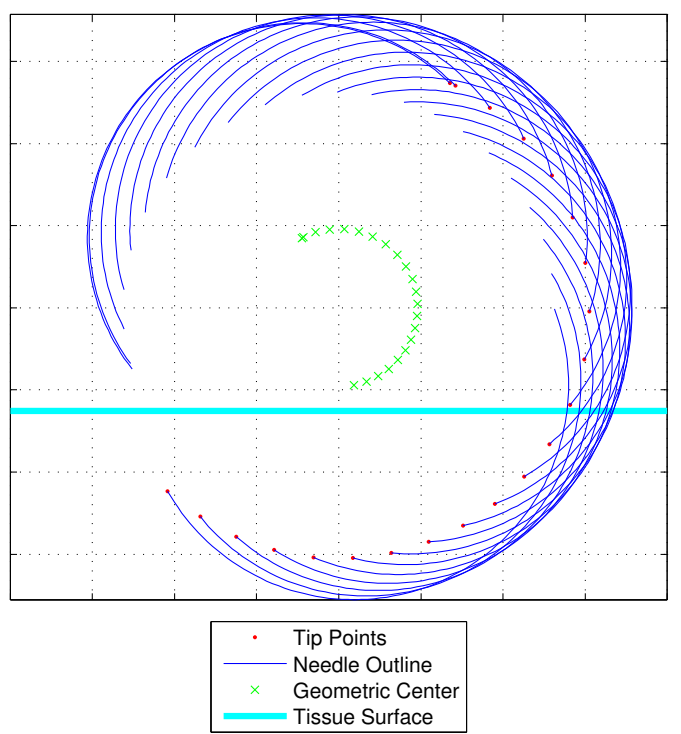

Fig. 4: Non-Ideal Area Sweep. The needle sweeps out an area in a clockwise direction during a suture. As the geometric center moves, the needle area sweep direction changes.

locally around the needle portion embedded in the tissue. The overall friction force becomes a sum of forces which are each acting in different directions. The friction force will then change significantly in magnitude and direction as the needle moves through the tissue.

\section{B. Area Forces}

When the needle moves in a non ideal fashion as in Fig. 2(b), it will press against the tissue as it moves. The area swept by the needle as calculated in (9) can be used as a basis for the normal force. If the tissue is treated as a Hookian material, then a spring constant $\mathbf{K}$ (measured in force per unit area) can be used to convert the area swept into a force magnitude. Since the tissue sample is typically not a cube, its spring constant may vary in different directions. Therefore $\mathbf{K}$ will be assumed to be a diagonal matrix instead of a scalar. Since the area computation includes both a magnitude and a direction, the tissue simply applies a restoring force to the needle. By modifying equation (8) to include $\mathbf{K}$, the normal force due to area can be computed

$$
\mathbf{f}_{n o r m a l}(\phi(t))=-\mathbf{K} \int_{\phi(0)}^{\phi(t)} \int_{\theta_{0}(\phi)}^{\theta_{1}(\phi)} \mathrm{d} \mathbf{a}_{n}(\phi, \theta) \mathrm{d} \theta \mathrm{d} \phi .
$$

\section{Cutting and Stiffness Forces}

The stiffness force models the forces applied by the needle to the tissue before the needle begins to penetrate the tissue. The force is modeled as the angle of entrance squared. When the stiffness force is larger than the cutting force, the needle is assumed to be cutting the tissue and the cutting force is used instead. Both forces act in opposition to the needle tip.

$$
\mathbf{f}_{\text {cutting }}(\phi(t))=-\min \left(\alpha,\left(\theta_{\text {tip }}-\theta_{s}\right)^{2} \beta\right) \mathrm{d} \ell\left(\theta_{\text {tip }}\right)
$$

The variable $\alpha$ is the maximum magnitude of the cutting force. The variable difference $\theta_{t i p}-\theta_{s}$ is the difference between the tissue intersection angle and the actual tip angle. $\beta$ is a scaling coefficient. $\mathrm{d} \ell\left(\theta_{\text {tip }}\right)$ is the tangent vector of the tip.

\section{Torque Calculations}

Since the needle is curved, torques can be an important indicator of the amount of tissue trauma that is occurring. The models are adapted to include modeling of the torques. The torques are computed using the following equations.

$$
\begin{gathered}
\tau_{\text {fric }}(\phi(t))=\int_{\theta_{0}(\phi)}^{\theta_{1}(\phi)}-\mu_{s} \mathbf{r}_{f}(\theta) \times \mathrm{d} \ell(\theta) \mathrm{d} \theta, \\
\tau_{\text {norm }}(\phi(t))= \\
-K \int_{\phi_{0}}^{\phi_{1}} \int_{\theta_{0}(\phi)}^{\theta_{1}(\phi)} \mathbf{r}_{f}(\theta) \times \mathrm{d} \mathbf{a}_{n}(\phi, \theta) \mathrm{d} \theta \mathrm{d} \phi, \\
\tau_{\text {cut }}(\phi(t))=\mathbf{r}_{f}\left(\theta_{\text {tip }}\right) \times \mathbf{f}_{\text {cutting }}(\phi(t)),
\end{gathered}
$$

where $\mathbf{r}_{f}$ is the vector from the force/torque sensor to the needle segment.

\section{RESULTS}

As part of this study, the proposed needle-tissue interaction force models were validated with experimental data collected using a circular robotic motor stage and a tissue phantom.

\section{A. Experimental Methods}

In the experiment, a custom made one degree-of-freedom (DOF) rotational motion stage equipped with a six DOF force/torque sensor (nano17 by ATI Industrial Automation, Apex, North Carolina) was used to drive a surgical suture needle into a tissue phantom. The interaction forces were recorded. The experimental setup is shown in Fig. 5. During the experiment, the motor turns the eccentrically mounted force sensor and needle such that the needle will drive through the tissue phantom. During the insertion, the motor turns with a constant velocity using a servo loop running at a $2 \mathrm{kHz}$ sampling rate. The tissue phantom used is a commercial training phantom that is a suture training aid for surgeons (SCS-10 by Simulab Corp., Seatle Washington). The tissue phantom has dimensions $105 \mathrm{~mm}$ by $105 \mathrm{~mm}$ by $20 \mathrm{~mm}$. It has two sides. One side simulates a layer of skin tissue. The other side simulates subcutaneous fat. The subcutaneous fat side was used during the experiment. The needle used in the experiment is a CT- 1 suture Needle (Ethicon Corp., Raleigh, NC). The needle is a half circle taper point needle that is $36 \mathrm{~mm}$ long. The needle is mounted such that the center of motion is offset from the geometric center of the needle so it is possible to measure the effects of non-ideal needle motion. For convenience of the reader and ease of interpretation, all of the force/torque measurements and models results are presented relative to the coordinate frame defined by $x_{f}$ and $y_{f}$ as shown in Fig. 1.

\section{B. Force Data Post Processing}

In order to be used for data analysis, the raw force torque data is post-processed. This removes three effects. The first effect is the force bias that the sensor naturally has $\left(\mathbf{f}_{\text {bias }}\right)$. 
The second correction is to rotate the force sensor frame so that it remains parallel to the global frame. The angle of rotation is $\rho$. The rotation of the needle center $\phi$ is included because the force sensor rotates with the needle. The final correction is to remove the effect of gravity on the needle $(m g)$. These corrections can be modeled as

$$
\begin{aligned}
\mathbf{f}_{\text {exp }} & =\left[\begin{array}{c}
0 \\
m g \\
0
\end{array}\right] \\
& +\left[\begin{array}{ccc}
\cos (\rho+\phi) & -\sin (\rho+\phi) & 0 \\
\sin (\rho+\phi) & \cos (\rho+\phi) & 0 \\
0 & 0 & 1
\end{array}\right]\left(\mathbf{f}_{\text {raw }}-\mathbf{f}_{\text {bias }}\right),
\end{aligned}
$$

where the raw force data is $\mathbf{f}_{r a w}$. The processed force data is $\mathbf{f}_{\text {exp }}$. To solve for all of the variables, the raw sensor data from when the needle is not penetrating the tissue is used. As the only force in free space acting on the needle is gravity, we would have

$$
\mathbf{f}_{\text {exp }}=\left[\begin{array}{l}
0 \\
0 \\
0
\end{array}\right] .
$$

This allows for the bias, the angle offset, and the gravity force to be estimated using the numerical minimization. The processed force and torque profiles are now only the result of needle tissue forces. The torque profiles are measured about the needle mount.

\section{Measured Force Data}

The results of 4 different experiments are plotted together in Fig. 6. The forces have been processed using (17). The plots are exclusively the needle tissue forces. The variability from one tissue run to the next is negligible. For brevity, the corresponding torque plots are not included. The forces felt by the needle in the $z$ direction are small compared to the $x$ and $y$ forces felt by the needle, therefore, the planar motion approximation is held. The magnitude of the measured forces is approximately $2.82 \mathrm{~N}$. This is similar in magnitude to the suture forces of a trained surgeon as measured by Dubrowski et al. [4].

\section{Parameter Fitting}

There are a total of 12 parameters that need to be determined to fit the model to the experimental data. These include the following variables: $M_{r}$ is the radius of motion of the needle holder and force sensor. $r_{c}, \kappa, r$ and $\phi_{0}$ are the geometric parameters all defined in Fig. 2(b). The arc length of the needle and the height of the tissue relative to the motor axis are the final geometric variables. The remaining variables are material variables, namely the friction coefficient $\mu_{s}$ as defined in (11), the spring constant matrix $\mathbf{K}$, and the $\alpha$ and $\beta$ parameters as defined in (13). The $\mathbf{K}$ matrix introduces two variables because only the $x$ and $y$ forces are influenced by the normal needle motion. The variables are estimated using using two methods. Some of the geometry can be directly measured from the system. This includes the tissue height relative to the motor axis and the radius of the

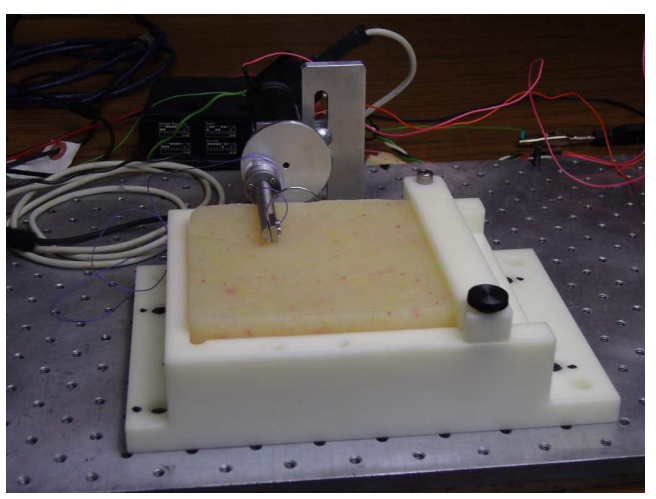

Fig. 5: Experimental Suture Apparatus. A motor holds a disc which mounts the needle base eccentrically. This allows the motor to turn the needle through an approximation of the ideal needle motion.
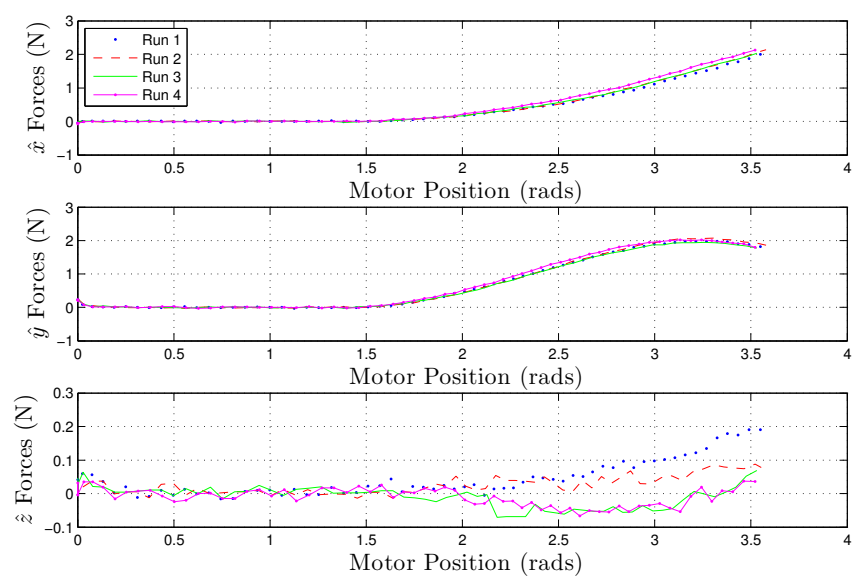

Fig. 6: Experimental Force Data. The plots display the results of four different needle drives. In order of descent, the plots show the $x, y$ and $z$ forces. The force measurements from multiple passes were similar. The measured $z$ forces are an order of magnitude smaller than the $x$ or $y$ forces.

motion of the needle mount. The remaining parameters were estimated simultaneously using a numerical minimization that matched the force model estimate with the experimental data. The numerical optimization method was implemented using MATLAB $^{\circledR}$. The linear force results are shown in Fig. 7 and the torques are in Fig. 8. The force breakdowns show the final contribution from each force and torque component in Fig. 9 and Fig. 10. Notice the normal and friction forces constructively add in the $y$ direction, but they destructively add for both the $x$ direction and the torques.

\section{CONClusions AND Future Work}

The results of the model fit closely match both the linear forces and the torques that the needle experiences during a suture. The lumped forces are a good approximation for canonical needle motion and small non ideal tests. It is important to note that the needle was moving slowly in the experiments so that the non viscous friction approximation would hold. In our future work, we als plan to collect 

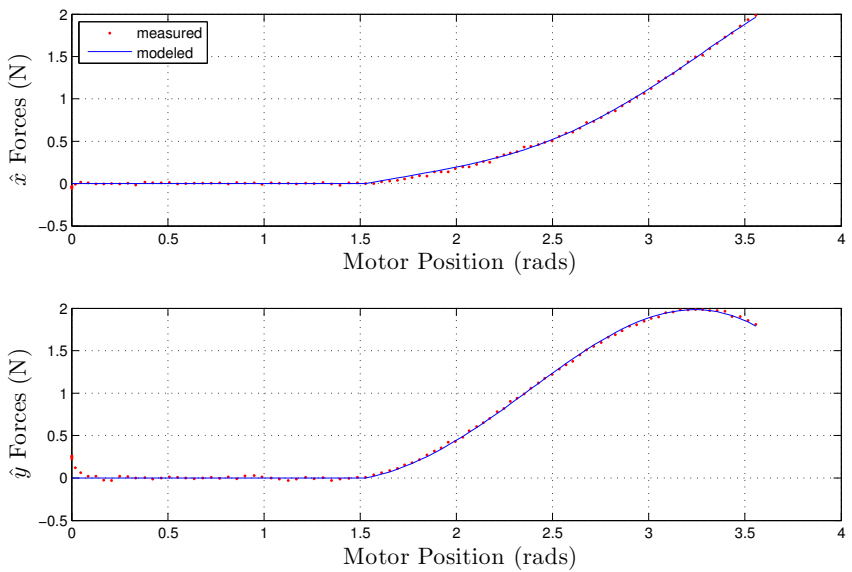

Fig. 7: The Linear Force Model. The experimental linear forces are plotted with both the model forces and the measured forces. The top plot is the $x$ direction and the bottom plot is the $y$ direction. Both sets of modeled forces closely track their measured counterparts.

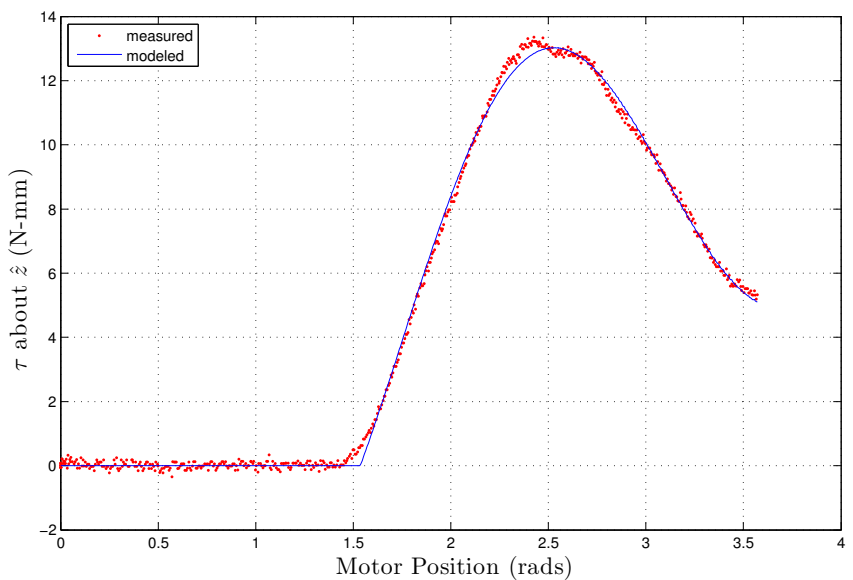

Fig. 8: The Torque Model. The torque model is plotted with the measured torque. There is strong correspondence between the two curves.

experimental data from ex vivo tissue samples in order to study the validity of the model for actual tissue. We are also planning to extend the model by relaxing the planar motion approximation. This modified model will be experimentally validated. A comparison between the lumped model and a tissue FEM model will also be pursued.

\section{REFERENCES}

[1] N. Abolhassani, R. Patel, and M. Moallem, "Needle insertion into soft tissue: A survey," Medical Engineering \& Physics, vol. 29, no. 4, pp. 413 - 431, 2007. [Online]. Available: http://www.sciencedirect.com/science/article/pii/S1350453306001457

[2] R. Alterovitz, K. Goldberg, J. Pouliot, and I.-C. Hsu, "Sensorless motion planning for medical needle insertion in deformable tissues," Information Technology in Biomedicine, IEEE Transactions on, vol. 13, no. 2, pp. $217-225$, march 2009.

[3] N. Chentanez, R. Alterovitz, D. Ritchie, L. Cho, K. K. Hauser, K. Goldberg, J. R. Shewchuk, and J. F. O'Brien, "Interactive simulation of surgical needle insertion and steering," in Proceedings of
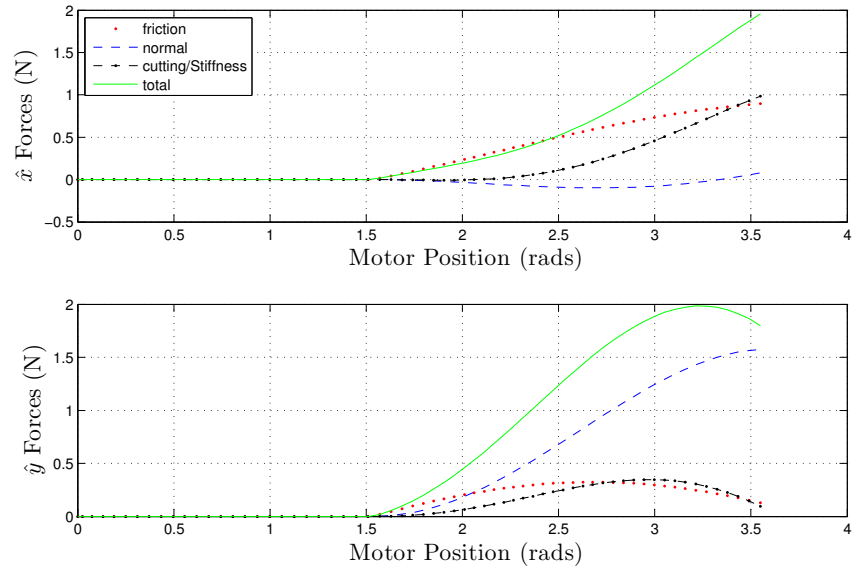

Fig. 9: The Linear Force Model Components. The force components $\mathbf{f}_{\text {friction }}, \mathbf{f}_{\text {normal }}$, and $\mathbf{f}_{\text {cutting }}$ are plotted with the total modeled force. The top plot is the $x$ direction and the bottom plot is the $y$ direction. All of the force components are significant relative to the total force.

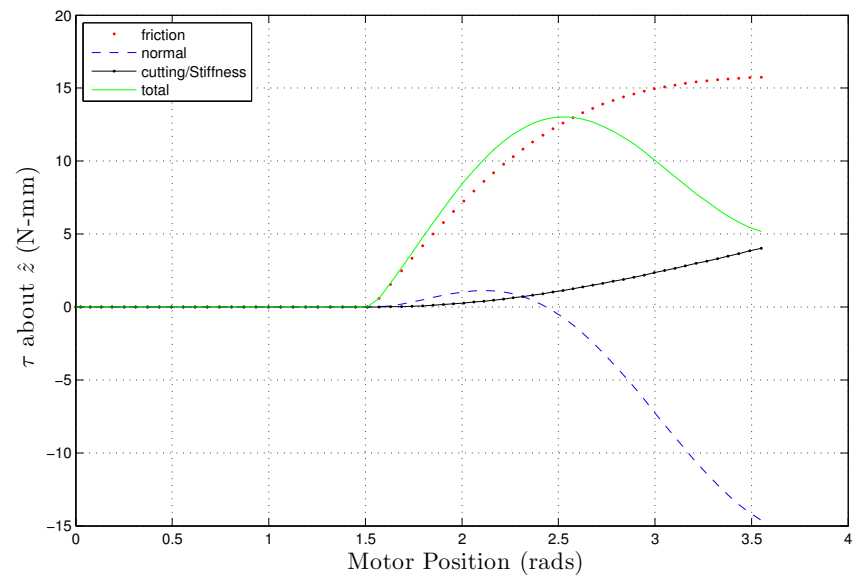

Fig. 10: The Torque Model Components. The torque components $\tau_{\text {friction }}, \tau_{\text {normal }}$, and $\tau_{\text {cutting }}$ are plotted with the total modeled torque. The torque due to the friction is positive while the torque from the normal force is negative.

ACM SIGGRAPH 2009, Aug 2009, pp. 88:1-10. [Online]. Available: http://graphics.berkeley.edu/papers/Chentanez-ISN-2009-08/

[4] A. Dubrowski, R. Sidhu, J. Park, and H. Carnahan, "Quantification of motion characteristics and forces applied to tissues during suturing," The American Journal of Surgery, vol. 190, no. 1 , pp. 131 - 136, 2005. [Online]. Available: http://www.sciencedirect.com/science/article/pii/S000296100500351X

[5] A. Maghsoudi and M. Jahed, "Multi-parameter sensitivity analysis for guided needle insertion through soft tissue," in Biomedical Engineering and Sciences (IECBES), 2010 IEEE EMBS Conference on, 30 2010-dec. 2 2010, pp. $97-100$.

[6] S. Misra, K. Reed, B. Schafer, K. Ramesh, and A. Okamura, "Observations and models for needle-tissue interactions," in Robotics and Automation, 2009. ICRA '09. IEEE International Conference on, may 2009, pp. $2687-2692$.

[7] A. Okamura, C. Simone, and M. O'Leary, "Force modeling for needle insertion into soft tissue," Biomedical Engineering, IEEE Transactions on, vol. 51, no. 10, pp. $1707-1716$, oct. 2004.

[8] D. A. Sherris and E. B. Kern, Essential Surgical Skills, 2nd ed. Saunders, 2004. 